\title{
Effects and mechanisms of Fenofibrate on the secretion of vascular endothelial contraction factors in hypertensive rats
}

\author{
Y. Zhu' ${ }^{1}$ H.-S. Wang ${ }^{1}$ X.-M. Li ${ }^{1}$ and C. $Q u^{2}$ \\ 'Department of Cardiac Surgery, \\ General Hospital of Shenyang Military Region, Shenyang, China \\ ${ }^{2}$ Department of Cardiology, \\ Shenyang Medical College Affiliated with Fengtian Hospital, Shenyang, China \\ Corresponding author: H.-S. Wang \\ E-mail: zhu13309888139@163.com
}

Genet. Mol. Res. 13 (3): 5269-5275 (2014)

Received April 25, 2013

Accepted November 1, 2013

Published July 24, 2014

DOI http://dx.doi.org/10.4238/2014.July.24.5

\begin{abstract}
This study investigated the effects of the peroxisome proliferator-activated receptor alpha (PPAR- $\alpha$ ) agonist, Fenofibrate, on the secretion of vascular endothelial contraction factors in hypertensive rats to elucidate its possible mechanisms. The vascular ring contraction experiment was used to observe whether rat vascular tension of clean grade spontaneously hypertensive rats (SHR) changes after 1-h incubation of $0.1,1.0,10.0 \mu \mathrm{M}$ Fenofibrate with $10.0 \mu \mathrm{M}$ Fenofibrate, a PPAR- $\alpha$ antagonist (MK866), and a PPAR- $\gamma$ antagonist (GW9662) in SHR. The results were compared with Wistar Kyoto rats. Enzyme-linked immunosorbent assay was used to detect the secretion of the serum vascular endothelial contraction factor prostacyclin-1 $\alpha$ (PGF-1 $\alpha$ ), PGF-2 $\alpha$, and thromboxane B2 (TXB2). Western blot was used to detect COX-1 protein expression. A quantity of $10.0 \mu \mathrm{M}$ Fenofibrate significantly reduced vasoconstriction in SHR compared to the control group $(\mathrm{P}=0.013)$. The PPAR- $\alpha$ antagonist, MK866, significantly improved the vascular contractility of SHR
\end{abstract}


when incubated with $10.0 \mu \mathrm{M}$ Fenofibrate $(\mathrm{P}=0.021)$. The PPAR- $\gamma$ antagonist, GW9662, had no significant effect on the vascular contractility of SHR when incubated with $10.0 \mu \mathrm{M}$ Fenofibrate $(\mathrm{P}=$ 0.071). The isolated aorta of SHR released significantly lower PGF$1 \alpha(\mathrm{P}=0.014), \mathrm{PGF}-2 \alpha(\mathrm{P}=0.023)$, and TXB2 $(\mathrm{P}=0.017)$ levels in the $10.0 \mu \mathrm{M}$ Fenofibrate group compared to the control group. COX-1 expression of SHR rat vascular endothelium was significantly depressed in the $10.0 \mu \mathrm{M}$ Fenofibrate group compared to the control group $(\mathrm{P}=0.027)$. In conclusion, Fenofibrate reduces the secretion of vascular endothelial contraction factors in hypertensive rats, which might arise through the endothelium influencing COX-1 expression.

Key words: Peroxisome proliferator-activated receptor $\alpha$; Fenofibrate; Vascular endothelial contraction factors; Cyclooxygenase

\section{INTRODUCTION}

The number of dyslipidemia patients is increasing annually because of economic development, social progress, and changes in dietary composition, leading to an escalation in the incidence of coronary heart disease in China. Studies have shown that decreased myocardial peroxisome proliferator-activated receptor $\alpha$ (PPAR- $\alpha$ ) expression might regulate myocardial substrate and energy metabolism (Karbowska et al., 2003). Therefore, the utilization of this PPAR- $\alpha$ role is expected to become a method of treatment and prevention in coronary heart disease (Berger et al., 2005). Lüscher and Vanhoutte (1986) demonstrated that the vascular endothelial contraction factor contributes to the occurrence and development of coronary heart disease. The presence of this factor risks the initiation of the vascular endothelium and atherosclerotic process. This sentence should be "Fenofibrate, a lipid-lowering drug, effectively improves the atherogenic lipid profile, which is associated with T2DM and MetS by activating PPAR- $\alpha$. In this study, we investigated the impact of the PPAR- $\alpha$ agonist, Fenofibrate, on the secretion of vascular endothelial cytokine in hypertensive rats to elucidate its possible mechanisms.

\section{MATERIAL AND METHODS}

\section{Materials}

This study used 40 male spontaneously hypertensive rats (SHR) of 38-42 weeks in age and 600-800 $\mathrm{g}$ in weight and 40 male WKY rats of 38-42 weeks in age and 300-400 $\mathrm{g}$ in weight. The rats were purchased from the Experimental Animal Center of the Chinese University of Hong Kong. Prostacyclin-1 $\alpha$ (PGF-1 $\alpha$ ), PGF-2 $\alpha$, thromboxane B2 (TXB2), enzyme-linked immunosorbent assay (ELISA) kit, goat anti-human endothelial nitric oxide synthase (eNOS), cyclooxygenase (COX), and antibody/rabbit anti-goat IgG antibodies were all purchased from the Cayman Chemical Company (USA). The Bio-Rad protein analyzer was purchased from the Amersham Pharmacia Biotech Company (Sweden). 


\section{Determination of aortic vascular tension in vitro}

The vascular ring contraction experiment was performed to detect isolated rat aortic tension. In brief, $70 \mathrm{mg}(\mathrm{mL} / \mathrm{kg})$ phenobarbital subcutaneous injection was used to anesthetize the rats. Ten minutes after blocking the blood flow from the aorta, a 3-mm long target vessel from the aorta was removed, and immediately stored in Krebs-Ringer bicarbonate buffer at $4^{\circ} \mathrm{C}$. When preparing the specimen, we carefully observed the wall of the target vessel to detect the presence of any artery atherosclerotic change or other visible disease. The acquired vascular ring was pulled taught with two iron wires and bathed in a $10-\mathrm{mL}$ tissue bath (at $37^{\circ} \mathrm{C}$, pH 7.4, filled with $95 \% \mathrm{O}_{2}$ and $5 \% \mathrm{CO}_{2}$ ). One end of the wire was connected to the tension sensor, transducer, amplifier, and multi-channel polygraph testing equipment to record changes in the isometric tension of the vascular ring. The other end of the wire was fixed to the fine adjustment equipment, for adjusting the passive tension. The entire device was washed at 15-min intervals with buffer. During this period, all vascular rings gradually attained the optimal resting tension. Continuous $2.5 \mathrm{~g}$ tension was given to the vascular rings until the end of the experiment. All experiments were completed on this experimental platform.

Twenty SHR tissues were randomly divided into the vascular endothelial group and non-vascular endothelial group, with 10 rats in each group. The rats were given Fenofibrate, the PPAR- $\alpha$ antagonist MK866, or the PPAR- $\gamma$ antagonist GW9662 and left for a 1-h incubation period (all the rats were given all three substances.). Twenty WKY rats served as controls. The drugs in the rat vascular ring contraction experiment were incubated with NG-nitro-Larginine methyl ester (L-NAME) at the same time, to exclude the interference of nitric oxide on the vascular endothelial contraction factor. Acetylcholine was given gradually $(0-10 \mathrm{mM})$ to stimulate vasoconstriction. Through comparison with a $60-\mathrm{mM}$ potassium chloride maximum contraction platform, a vascular ring contraction curve was drawn. The curve changed at different concentrations and with different drugs. All values are reported as percentages.

\section{Determination of vascular endothelial contraction factor}

According to the above method, when the vasoconstriction effect was at a maximum, we collected the Kreb's liquid and stored it at $-80^{\circ} \mathrm{C}$ for further analysis. PGF- $1 \alpha$, PGF-2 $\alpha$, and TXB2 concentrations were measured by ELISA.

\section{COX-1 protein detection}

The artery rings were continuously incubated for $24 \mathrm{~h}$ after the experiment, the vascular tissue and the frozen extraction buffer $(10 \mathrm{mM}$ Tris- $\mathrm{HCl}+140 \mathrm{mM} \mathrm{NaCl}+1 \mathrm{mM}$ EDTA $+25 \%$ glycerol $+0.5 \%$ SDS $+0.5 \%$ Nonident P-40 + $0.1 \mathrm{mM} \mathrm{PMSF}+100 \mathrm{ng} / \mathrm{mL}$ protease inhibitor cocktail, pH7.5, 1:3 w/v) were homogenized (2000 rpm, $30 \mathrm{~s})$. The rings were centrifuged at $14,000 \mathrm{rpm}$ at $4^{\circ} \mathrm{C}$ for $10 \mathrm{~min}$. The Bio-Rad protein analyzer was used to measure supernatant protein concentrations. Then, the rings were frozen in liquid nitrogen and stored at $-80^{\circ} \mathrm{C}$. A volume of $40 \mu \mathrm{g}$ blood that had been extracted from the cytoplasm protein was added to the sodium dodecyl sulfate solution at $95^{\circ}-100^{\circ} \mathrm{C}$ for $5 \mathrm{~min}$. Then, $10 \%$ sodium dodecyl sodium phosphate gel electrophoresis was used to transfer the proteins to the nitrocellulose membrane. The proteins were placed at the top of blocking solution [5\% skimmed milk dis- 
solved in Tris-HCl-buffered saline and Tween (TBST)] for $2 \mathrm{~h}$. Then, goat anti-human eNOS and COX antibody, which had been diluted in TBST (containing $5 \%$ bovine serum albumin), were added at a ratio of 1:400 and kept at $4{ }^{\circ} \mathrm{C}$ overnight. The horseradish peroxidase-labeled rabbit anti-goat IgG antibody, which had been diluted in TBST (containing $5 \%$ bovine serum albumin), was added at a ratio of 1:2000. After 1.5-2 $\mathrm{h}$ at room temperature, the 1:20 diluted peroxide and LumiGLO ${ }^{\mathrm{TM}}$ chemiluminescent agents were reacted for $1 \mathrm{~min}$. Then, the results were automatically obtained by radiography.

\section{Statistical analysis}

The SPSS 14.0 statistical software was used for all analyses. The experimental data are reported as means \pm standard deviation. The $t$-test was used to compare differences between the groups. Significant difference was set at $\mathrm{P}<0.05$.

\section{RESULTS}

\section{Aortic vascular tension measurements in vitro}

Nitric oxide synthase caused the vascular rings of control rats to gradually contract as the concentration of acetylcholine increased. The highest vascular ring contraction was recorded at acetylcholine concentrations of 10-4 mM (82.0 $\pm 2.0 \%)$. After 1-h incubation with 0.1 and $1.0 \mu \mathrm{M}$ Fenofibrate and at acetylcholine concentrations of $10-4 \mathrm{mM}$, the degree of vasoconstriction in SHR was $81.0 \pm 1.1$ and $80.0 \pm 3.0 \%$. This difference was not statistically significant compared to the control group $(\mathrm{P}=0.013)$. At $1 \mathrm{~h}$ after incubation with $10.0 \mu \mathrm{M}$ Fenofibrate, the degree of vasoconstriction in SHR was $27.0 \pm 1.9 \%$, which was significantly lower than the control group $(\mathrm{P}=0.013$; Figure 1). After 1-h incubation of $10.0 \mu \mathrm{M}$ Fenofibrate and the PPAR- $\alpha$ antagonist MK866, the degree of vasoconstriction in SHR was 78.0 \pm $3.0 \%$, which was significantly higher compared to the $10.0 \mu \mathrm{M}$ Fenofibrate incubation group $(\mathrm{P}=0.021)$. After 1-h incubation of $10.0 \mu \mathrm{M}$ Fenofibrate and the PPAR- $\gamma$ antagonist GW9662, the degree of vasoconstriction in SHR showed no statistically significant difference to that of the $10.0 \mu \mathrm{M}$ Fenofibrate incubation group $(\mathrm{P}=0.071$; Figure 2$)$.

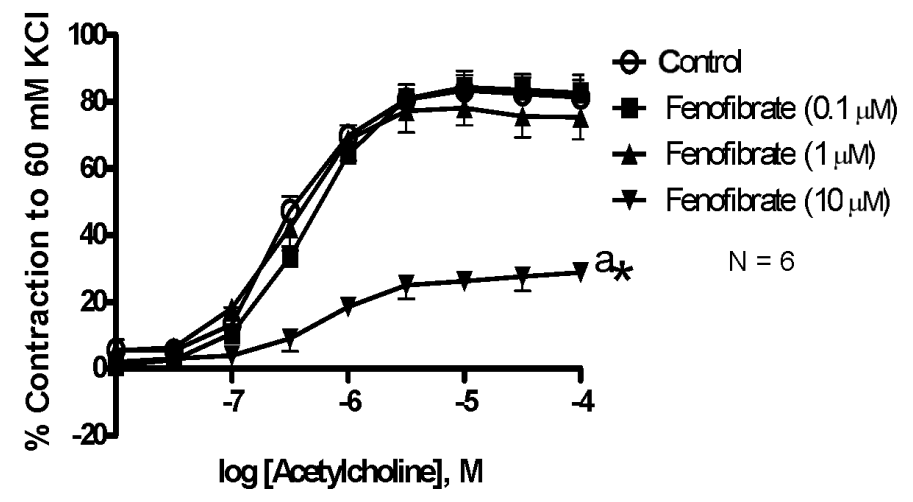

Figure 1. Effect of Fenofibrate on vascular tension. $\mathrm{P}=0.013$ compared with the control group. 


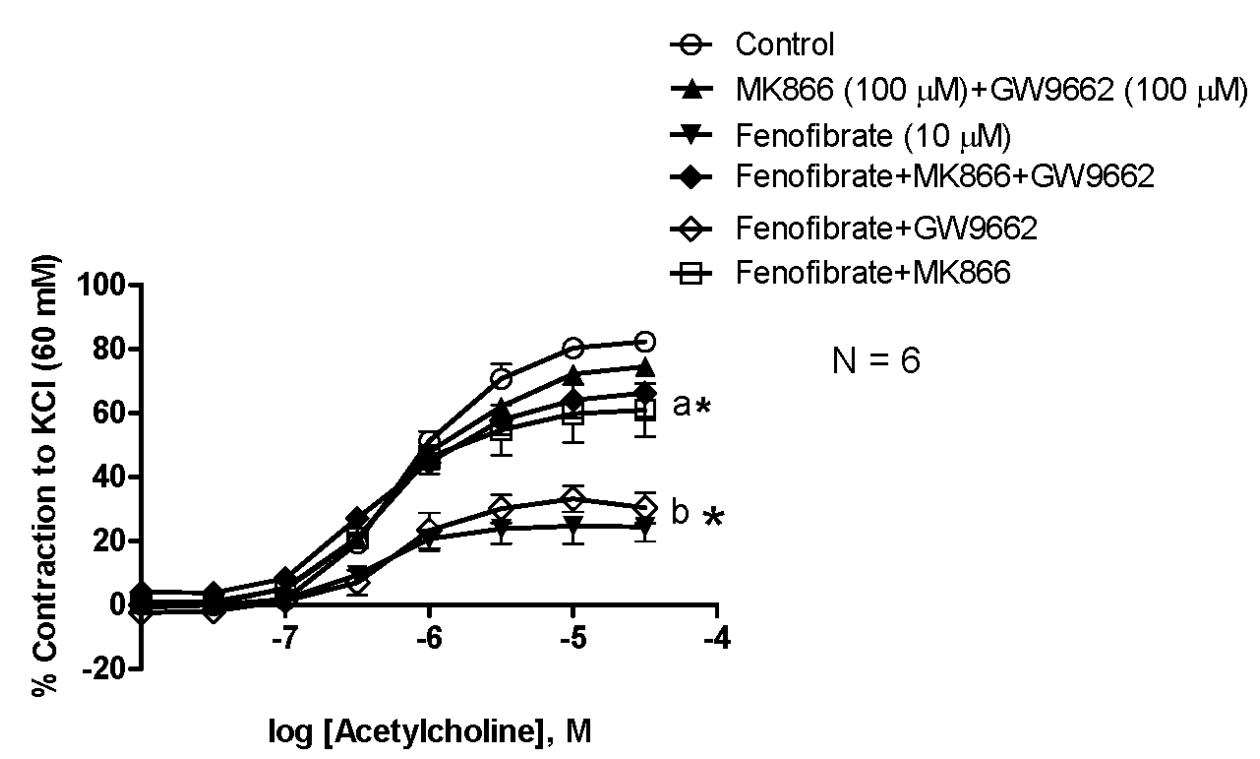

Figure 2. Effect of cyclooxygenase antagonist on vascular tension. $* \mathrm{P}=0.021$ and $* \mathrm{P}=0.071$ compared with the 10.0 $\mu$ M Fenofibrate incubation group. $\mathrm{a}=$ MK866; b = GW9662.

\section{Secretion of vascular endothelial constraction factor}

Compared with the non-Fenofibrate treatment group, the PGF-1 $\alpha$, PGF- $2 \alpha$ and TXB2 levels in the $10 \mu \mathrm{M}$ Fenofibrate group significantly decreased $(\mathrm{P}=0.014, \mathrm{P}=0.023, \mathrm{P}=0.017$, respectively; Table 1).

\begin{tabular}{|c|c|c|c|}
\hline Group & PGF-1 $\alpha$ & PGF- $2 \alpha$ & TXB2 \\
\hline Non-Fenofibrate group & $34,212 \pm 2113$ & $17,746 \pm 1009$ & $3333 \pm 234$ \\
\hline $10 \mu \mathrm{M}$ Fenofibrate group & $1804 \pm 186$ & $228 \pm 57$ & $119 \pm 21$ \\
\hline$P$ value & 0.014 & 0.023 & 0.017 \\
\hline
\end{tabular}

Data are reported as means $\pm \mathrm{SD}$ in $\mathrm{pg} / \mathrm{mL}$ for 6 rats in each group.

\section{COX-1 expression}

Compared to the SHR in the non-Fenofibrate treatment group, after incubation with Fenofibrate for $24 \mathrm{~h}$, the rats with the vascular endothelium exhibited significantly lower COX-1 expression $(\mathrm{P}=0.027)$. COX-1 expression did not change significantly in rats that did not have the vascular endothelium $(\mathrm{P}=0.074)$. After 24-h Fenofibrate incubation, COX-1 expression of rats with and without the vascular endothelium in the WKY group did not show any significant difference to rats in the non-Fenofibrate incubation group $(\mathrm{P}=0.086$ and $\mathrm{P}=$ 0.067, respectively; Figure 3). 

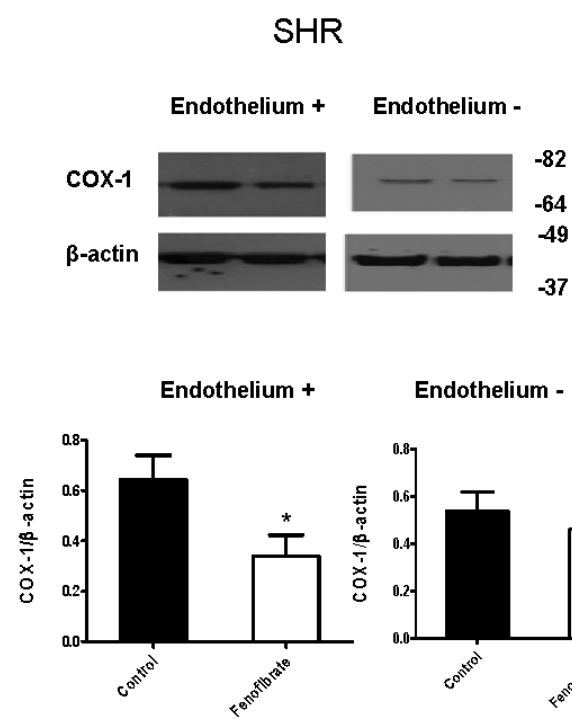
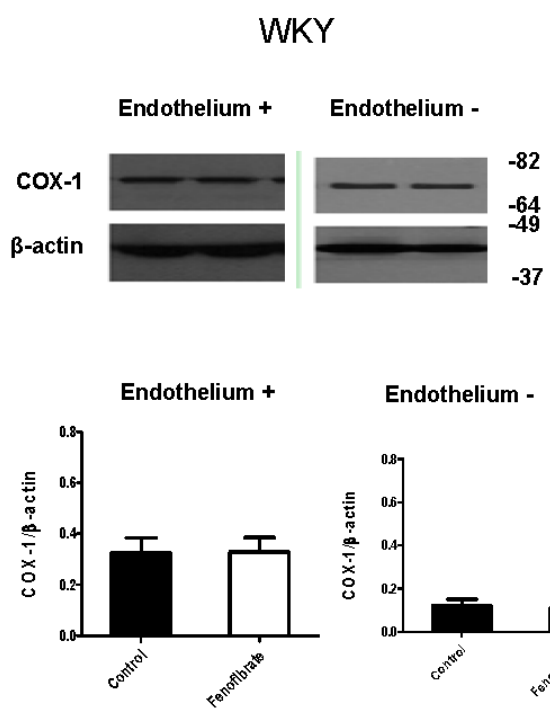

Endothelium -



Figure 3. Expression of cyclooxygenase-1. $* \mathrm{P}=0.027$ compared with the control group.

\section{DISCUSSION}

The vascular endothelium is an important structure for the regulation of vascular tension. This tissue regulates vascular contraction through the release of vascular endothelial contraction factors and diastolic factors. Vascular endothelial dysfunction is an important initiating factor leading to cardiovascular and cerebrovascular diseases. In certain pathological conditions, such as the hypertension, coronary heart disease, and endothelial function impairment, the vascular endothelium releases lower quantities of dilating factors, and causes vasoconstriction, leading to the further aggravation of the disease (Auch-Schwelk et al., 1992). This leads to the formation of a vicious circle between the disease and the causative factors. Yang et al. (2004) found that the increased release of vascular endothelial contraction factor is a major contributor to endothelial injury. Vascular endothelial contraction factors perform antagonistic functions to the dilating factors. These contraction factors have opposing contracting and dilating effects on endothelial vascular smooth muscle (Mombouli and Vanhoutte, 1999; Tang et al., 2005a; Vanhoutte et al., 2009). The main purpose of this study was to investigate the effects of Fenofibrate on vascular endothelial contraction factors. In the isolated aortic vascular tension determination experiment, all experimental drugs were incubated in combination with the nitric oxide synthase inhibitor, L-NAME, to exclude the interference of nitric oxide (main vascular dilating factor in SHR), and to amplify the release of vascular endothelial contraction factor. The results showed that after $1 \mathrm{~h}$ of $10.0 \mu \mathrm{M}$ Fenofibrate incubation, the vascular contractility of SHR significantly decreased compared to the control group. This result indicates that Fenofibrate significantly alleviates the vasoconstriction state. Therefore, the current study confirmed the effects of Fenofibrate on the release vascular contraction factors in SHR. The results showed that compared with the non-Fenofibrate group, the isolated aortic tissue of SHR released significantly less PGF- $1 \alpha$, PGF- $2 \alpha$, and TXB2 in the $10 \mu \mathrm{M}$ Fenofibrate group. This 
result indicates that Fenofibrate might reduce the release of vasoconstrictor factors to reduce the vasoconstriction status.

It is known that the production of vascular endothelial contraction factors is mediated by COX. Previous studies have found that, in SHR, COX-1 is the key enzyme mediating vascular endothelial contraction factor (Yang et al., 2003; Tang et al., 2005b). The current study also observed the effects of Fenofibrate on COX-1 expression, in the presence and absence of endothelium, by Western blot assay. The results confirmed that in the presence of vascular endothelium, Fenofibrate treatment reduces COX-1 expression in rats. These results indicate that the COX-1 subtype is responsible for the effects of Fenofibrate on cyclooxygenase.

In conclusion, this study showed that the lipid-lowering drug Fenofibrate might improve vascular endothelial contraction and reduce the secretion of deleterious vascular endothelial contraction factors, such as PGF-1 $\alpha$, PGF- $2 \alpha$, and TXB2. This effect might be achieved by reducing COX-1 expression.

\section{REFERENCES}

Auch-Schwelk W, Katusic ZS and Vanhoutte PM (1992). Nitric oxide inactivates endothelium-derived contracting factor in the rat aorta. Hypertension 19: 442-445.

Berger JP, Akiyama TE and Meinke PT (2005). PPARs: therapeutic targets for metabolic disease. Trends Pharmacol. Sci. 26: $244-251$

Karbowska J, Kochan Z and Smolenski RT (2003). Peroxisome proliferator-activated receptor alpha is downregulated in the failing human heart. Cell. Mol. Biol. Lett. 8: 49-53.

Lüscher TF and Vanhoutte PM (1986). Endothelium-dependent contractions to acetylcholine in the aorta of the spontaneously hypertensive rat. Hypertension 8: 344-348.

Mombouli JV and Vanhoutte PM (1999). Endothelial dysfunction: from physiology to therapy. J. Mol. Cell. Cardiol. 31: 61-74.

Tang EH, Feletou M, Huang Y, Man RY, et al. (2005a). Acetylcholine and sodium nitroprusside cause long-term inhibition of EDCF-mediated contractions. Am. J. Physiol. Heart Circ. Physiol. 289: H2434-H2440.

Tang EH, Ku DD, Tipoe GL, Feletou M, et al. (2005b). Endothelium-dependent contractions occur in the aorta of wildtype and COX2 ${ }^{-/}$knockout but not COX1 ${ }^{-/}$knockout mice. J. Cardiovasc. Pharmacol. 46: 761-765.

Vanhoutte PM, Shimokawa H, Tang EH and Feletou M (2009). Endothelial dysfunction and vascular disease. Acta Physiol. 196: 193-222.

Yang D, Feletou M, Levens N, Zhang JN, et al. (2003). A diffusible substance(s) mediates endothelium-dependent contractions in the aorta of SHR. Hypertension 41: 143-148.

Yang D, Gluais P, Zhang JN, Vanhoutte PM, et al. (2004). Nitric oxide and inactivation of the endothelium-dependent contracting factor released by acetylcholine in spontaneously hypertensive rat. J. Cardiovasc. Pharmacol. 43: 815-820. 